\title{
Time-dependent Analysis of Optical Receivers Using Receiver Eigenmodes
}

\author{
Kyung Hee Seo ${ }^{1}$, Jae Seung Lee ${ }^{1 *}$, and Alan E. Willner ${ }^{2}$ \\ ${ }^{l}$ Department of Electronic Engineering, Kwangwoon University, Kwangwoon-ro 20, Nowon-gu, \\ Seoul 139-701, Korea \\ ${ }^{2}$ Department of Electrical Engineering, University of Southern California, \\ Los Angeles, California 90089, USA
}

(Received May 7, 2013 : revised June 26, 2013 : accepted June 27, 2013)

\begin{abstract}
Using receiver eigenmodes, we perform a time-dependent analysis of optical receivers whose optical inputs are corrupted by the amplified spontaneous emission. We use Gaussian receivers for the analysis with Gaussian input pulses. We find the number of contributing eigenmodes increases as the measurement time moves from the pulse center towards the pulse edges at the output of the optical receiver's electrical filter. This behavior is dependent on the bandwidth ratio between the optical and the electrical filters as well as the input pulse's time width.
Keywords: Optical communication, Optical modulation, Optical receivers, Optical amplifiers, Gaussian optical receiver

OCIS codes : (060.2330) Fiber optics communications; (060.2340) Fiber optics components; (060.4080) Modulation; (060.4510) Optical communications
\end{abstract}

\section{INTRODUCTION}

Optical receivers are basic building blocks in various kinds of optical communication systems. To increase the transmission distances, optical receivers suffer from various electrical and optical noises. In early optical communication systems, shot and thermal noises limit the receiver performances dominantly [1,2]. After the appearance of optical amplifiers, the amplified-spontaneous emission (ASE) becomes the most dominant noise source that mixes with the optical channel at the photo-detector [3-5]. For the analysis of optical receivers in the presence of the ASE, there are exact methods using receiver eigenmodes in the time domain [6-9] and in the optical spectral domain [10-13]. The time domain analyses have been developed initially for radiofrequency receivers.

Conventional analyses find the receiver eigenmode contributions at a specific time where the decision is made. Actually, the receiver eigenmode contributions change as a function of time within a bit period. These behaviors are affected by the optical and the electrical filters within the optical receiver. Accordingly, the distribution of the electrical voltage after the electrical filter will undergo similar changes within a pulse. Until now, however, no time-dependent analysis has been performed yet which is important for understanding the physics of optical receivers.

In this paper, we provide a time-dependent analysis of optical receivers whose optical inputs are corrupted by the ASE. We use the receiver eigenmodes in the optical spectral domain. Our analysis explains the physics about the receiver output signal and noise distributions as a function of time using receiver eigenmodes. We choose Gaussian optical receivers [14] that are good approximations to practical optical receivers yielding quantitative results with closed-form eigenfunctions.

\section{TIME-DEPENDENT OPTICAL RECEIVER ANALYSYS}

We assume the optical receiver has an optical filter in front of a photo-detector to select an optical channel and also to filter out the ASEs from optical amplifiers. After the photo-detector, there is a low-pass electrical filter to filter out high frequency electrical beat noises.

For simplicity, we neglect the polarization component

\footnotetext{
*Corresponding author: jslee@kw.ac.kr

Color versions of one or more of the figures in this paper are available online.
} 
perpendicular to that of the received signal. It will be included as an additive term after the signal contributions are fully evaluated. Just before the optical filter, the complexelectric-field amplitude is denoted as $\varepsilon_{i n}(\omega)$ in the optical frequency domain. After the optical filter, the complexelectric-field amplitude is $\varepsilon_{o u t}(\omega)=\varepsilon_{\text {in }}(\omega) H_{o}(\omega)$, where $H_{o}(\omega)$ is the optical filter's transmittance for the complex-electricfield amplitude. After the optical detection, the photo-detector output current is $(k / 2)\left|E_{\text {out }}(t)\right|^{2}$, where $k$ is a proportional constant and $E_{\text {out }}(t)$ is the inverse Fourier transform of $\varepsilon_{\text {ou }}(\omega)$. After the electrical filter having a transfer function of $H_{e}(\omega)$, we have the voltage waveform at the output of the electrical filter as

$$
y(t)=\frac{k}{8 \pi^{2}} \int_{-\infty}^{\infty} d \omega \int_{-\infty}^{\infty} d \omega^{\prime} \varepsilon^{*}{ }_{i n}(\omega) K\left(\omega, \omega^{\prime}\right) \varepsilon_{i n}\left(\omega^{\prime}\right) \exp \left\{j\left(\omega^{\prime}-\omega\right) t\right\},
$$

where the kernel $K\left(\omega, \omega^{\prime}\right)$ is given by

$$
K\left(\omega, \omega^{\prime}\right)=H_{o}^{*}(\omega) H_{e}\left(\omega^{\prime}-\omega\right) H_{o}\left(\omega^{\prime}\right) .
$$

We expand $\varepsilon_{i n}(\omega) \exp (j \omega t)$ in (1) as

$$
\varepsilon_{i n}(\omega) \exp (j \omega t)=\sum_{m=0}^{\infty} V_{m}(t) \phi_{m}(\omega),
$$

where $\phi_{m}(\omega)$ is the eigenfunction of the $m$-th receiver eigenmode and $V_{m}(t)$ is its expansion coefficient. The eigenfunctions satisfy the second kind of homogeneous Fredholm integral equation

$$
\phi_{m}(\omega)=\lambda_{m} \int_{-\infty}^{\infty} d \omega^{\prime} K\left(\omega, \omega^{\prime}\right) \phi_{m}\left(\omega^{\prime}\right) .
$$

$\lambda_{m}$ is the eigenvalue of the $\mathrm{m}$-th receiver eigenmode. The eigenvalues are real since the kernel is Hermitian. The eigenfunctions satisfy the orthonormal relation $\int_{-\infty}^{\infty} d \omega \phi_{m}(\omega)$ $\phi_{n}^{*}(\omega)=\delta_{m n}$, where the asterisk symbol represents the complex conjugation.

Using (3) for (1), $y(t)$ can be simplified greatly as a summation of receiver eigenmode contributions

$$
y(t)=\frac{k}{8 \pi^{2}} \sum_{m=0}^{\infty} \frac{\left|V_{m}(t)\right|^{2}}{\lambda_{m}} .
$$

The amplitude of the $m$-th eigenmode $V_{m}(t)$ can be decomposed into signal and noise components denoted as $S_{m}(t)$ and $N_{m}(t)$, respectively, as

$$
V_{m}(t)=S_{m}(t)+N_{m}(t) .
$$

Without loss of generality, we may set $t=0$ in (3). Then we have

$$
\varepsilon_{i n}(\omega)=\sum_{m=0}^{\infty} V_{m}(0) \phi_{m}(\omega) .
$$

The inverse Fourier transform of (7) gives

$$
E_{i n}(t)=\frac{1}{\sqrt{2 \pi}} \sum_{m=0}^{\infty} V_{m}(0) \psi_{m}(t)
$$

where $\psi_{m}(t)$ is $\sqrt{2 \pi}$ times the inverse Fourier transform of $\phi_{m}(\omega)$ and also satisfies the orthonormal relation in time domain $\int_{-\infty}^{\infty} d t \psi_{m}(t) \psi_{n}^{*}(t)=\delta_{m n}$.

Inserting (7) into (3), we find

$$
\sum_{m=0}^{\infty} V_{m}(t) \phi_{m}(\omega)=\sum_{k=0}^{\infty} V_{k}(0) \phi_{k}(\omega) \exp (j \omega t) .
$$

From this relation, $V_{m}(t)$ is found as

$$
\begin{aligned}
V_{m}(t) & =\sum_{k=0}^{\infty} V_{k}(0) \int_{-\infty}^{\infty} d \omega \phi_{k}(\omega) \phi_{m}^{*}(\omega) \exp (j \omega t) \\
& =\sum_{k=0}^{\infty} V_{k}(0) \int_{-\infty}^{\infty} d \tau \psi_{k}(\tau) \psi_{m}^{*}(\tau-t) \\
& =\sum_{k=0}^{\infty} V_{k}(0) C_{k m}(t)
\end{aligned}
$$

where $C_{k m}(t)$ is a correlation function

$$
C_{k m}(t)=C_{m k}^{*}(-t)=\int_{-\infty}^{\infty} d \tau \psi_{k}(\tau) \psi_{m}^{*}(\tau-t) .
$$

Equation (12) shows that the expansion coefficient set $\left\{V_{m}(t)\right\}$ at $t \neq 0$ can be found from $\left\{V_{m}(0)\right\}$ with $m=0,1,2 \ldots$

Multiplying both sides of (3) with their complex conjugates and integrating over $\omega$, we obtain [14]

$$
\int_{-\infty}^{\infty}\left|\varepsilon_{i n}(\omega)\right|^{2} d \omega=\sum_{m=0}^{\infty}\left|V_{m}(t)\right|^{2}
$$

Equating the signal parts from both sides of (14), we have

$$
\int_{-\infty}^{\infty}\left|\varepsilon_{i n, s}(\omega)\right|^{2} d \omega=\sum_{m=0}^{\infty}\left|S_{m}(t)\right|^{2}
$$

where $\varepsilon_{i n, s}(\omega)$ is the signal part of $\varepsilon_{i n}(\omega)$. It means that the sum of $\left|S_{m}(t)\right|^{2}$ is a constant proportional to the total signal energy assumed to be finite here. Note that (15) holds independent of time. Thus, if the amplitude of a dominant eigenmode decreases, the amplitudes of other eigenmodes 
increase making the sum of $\left|S_{m}(t)\right|^{2}$ over m unchanged.

If the other polarization component of the received channel is included, (5) may be rewritten as

$$
y(t)=\frac{k}{8 \pi^{2}} \sum_{m=0}^{\infty} \frac{\left|V_{m}(t)\right|^{2}+\left|V_{m}^{\prime}(t)\right|^{2}}{\lambda_{m}},
$$

where we have used a prime notation for the contributions from the newly added polarization component. The polarization vector for the second term of (16) has only noise components and $V_{m}^{\prime}(t)=N_{m}^{\prime}(t)$. All the real and imaginary parts of $\left\{N_{m}(t)\right\}$ and $\left\{N_{m}^{\prime}(t)\right\}$ at a fixed time are mutually independent Gaussian random variables with zero mean and an identical variance of $\sigma_{\omega}^{2}[10]$.

In dense wavelength-division multiplexing (DWDM) systems $[15,16]$, the optical filter's bandwidth is comparable to the modulation bandwidth. In this case, the magnitude of the eigenvalue $\lambda_{m}$ increases rapidly as $\mathrm{m}$ increases from zero $[10,14]$. This can be understood by the decrease in the freedom of mixing between optical frequency components in DWDM systems. As a result, the first term of (16) will be dominant as long as $\left.\left|S_{0}(t)\right|>\right\rangle\left|S_{m}(t)\right|$ for $m \neq 0$ such that

$$
y(t) \approx \frac{k}{8 \pi^{2} \lambda_{0}}\left\{\left|S_{0}(t)+N_{0}(t)\right|^{2}+\left|N_{0}^{\prime}(t)\right|^{2}\right\} .
$$

If we use $\psi_{0}(t)$ for a single mark signal transmission, it means $V_{m}(0)=0$ for $m \neq 0 . \psi_{0}(t)$ has a single maximum and the corresponding lowest-order eigenfunction in the spectral domain $\phi_{0}(\omega)$ occupies the smallest bandwidth of all eigenfunctions. We can prove that, in this case, the signal-to-noise ratio is maximized at $t=0$ [14] and the decision time should be chosen here to have the voltage

$$
y(0)=\frac{k}{8 \pi^{2}}\left\{\frac{\left|S_{0}(0)+N_{0}(0)\right|^{2}+\left|N_{0}^{\prime}(0)\right|^{2}}{\lambda_{0}}+\sum_{m=1}^{\infty} \frac{\left|N_{m}(0)\right|^{2}+\left|N_{m}^{\prime}(0)\right|^{2}}{\lambda_{m}}\right\} .
$$

In DWDM systems, (18) may be approximated as

$$
y(0) \approx \frac{k}{8 \pi^{2}}\left\{\frac{\left|S_{0}(0)+N_{0}(0)\right|^{2}+\left|N_{0}^{\prime}(0)\right|^{2}}{\lambda_{0}}\right\} .
$$

It is straightforward that the probability distribution function (pdf) of $y(0)$ from (19) has a non-central chi-square distribution $[17,18]$. As $|t|$ increases from zero, higher-order receiver eigenmode contributions to $y(t)$ increase from the relation $S_{m}(t)=S_{0}(0) C_{0 m}(t)$. At the same time, the magnitude of $S_{0}(t)$ decreases since the total sum of $\left|S_{m}(t)\right|^{2}$ is conserved as $\left|S_{0}(0)\right|^{2}=\sum_{m=0}^{\infty}\left|S_{m}(t)\right|^{2}$. Therefore, according to the central-limit theorem [19], the pdf of $y(t)$ becomes closer to Gaussian as the number of non-negligible or contributing eigenmodes increases. Note that the pdf of $y(0)$ becomes more symmetric also when its mean value increases. If we use pulses somewhat different from $\psi_{0}(t)$ to send a mark signal, normally a small number of eigenmodes would contribute to $y(0)$ from the lowest order and the pdf of $y(t)$ would resemble the Gaussian more closely. These behaviors can be explained more quantitatively using Gaussian optical receivers.

\section{ANALYSIS USING GAUSSIAN OPTICAL RECEIVERS}

We use Gaussian optical receivers [14] for our time-dependent analysis since they are good approximations to various optical receivers and have closed form eigenfunctions. We will show that, when the received optical pulse is a Gaussian pulse, the electrical pulse after the electrical filter is also a Gaussian pulse. Then our results will be compared with our foregoing time-dependent analysis.

The optical filter of a Gaussian optical receiver has a Gaussian impulse response as

$$
h_{o}(t)=A_{o} \exp \left\{\alpha^{2}\left(t-t_{o}\right)^{2} / 2\right\},
$$

where $t_{o}$ is the time delay of the optical filter. $h_{0}(t)$ is the inverse Fourier transform of $H_{o}(\omega)$. Also, the electrical filter of a Gaussian optical receiver has a Gaussian impulse response

$$
h_{e}(t)=A_{e} \exp \left\{\beta^{2}\left(t-t_{e}\right)^{2} / 2\right\},
$$

where $t_{e}$ is the time delay of the electrical filter. The 3-dB bandwidth of $\left|H_{o}(\omega)\right|^{2}$ is $\sqrt{\ln 2} \alpha / \pi$ from $H_{o}(\omega)=\sqrt{2 \pi}\left(A_{o} / \alpha\right) \exp$ $\left(-j \omega t_{o}-\omega^{2} / 2 \alpha^{2}\right)$. Similarly, the 3-dB bandwidth of $\left|H_{e}(\omega)\right|^{2}$ measured from the origin is $\sqrt{\ln 2} \beta / 2 \pi$.

The Gaussian receiver's eigenfunction $\phi_{m}(\omega)$ is given as a Hermite function times $\exp \left(j \omega t_{d}\right)$ as follows:

$$
\phi_{m}(\omega)=\frac{\exp \left(j \omega t_{d}\right)}{\sqrt{\sqrt{\pi} m ! 2^{m} a}} H_{m}\left(\frac{\omega}{a}\right) \exp \left(-\frac{\omega^{2}}{2 a^{2}}\right),
$$

where, $t_{d}\left(=t_{o}+t_{e}\right)$ is the total time delay of the receiver. $H_{m}(\omega / a)$ is the Hermite polynomial. The $3-\mathrm{dB}$ bandwidth of $\left|\phi_{0}(\omega)\right|^{2}$ is $\sqrt{\ln 2} a / \pi$. The parameters, $a, \alpha$, and $\beta$, are related as $\alpha^{2}=a^{2}(1+q) /(1-q)$ and $\beta^{2}=a^{2}\left(1-q^{2}\right) / 2 q$, where $q$ is a positive quantity less than 1 given by

$$
q=1+\frac{4}{r^{2}}\left(1-\sqrt{1+\frac{r^{2}}{2}}\right) .
$$

$r=2 \alpha / \beta$ is the $3-\mathrm{dB}$ bandwidth ratio of the optical and the 
electrical filters. Note that the Fourier transform of the Hermite function is also proportional to a Hermite function. Thus we have

$$
\psi_{m}(t)=j^{m} \sqrt{\frac{a}{\sqrt{\pi} m ! 2^{m}}} H_{m}\left\{a\left(t+t_{d}\right)\right\} \exp \left\{-\frac{a^{2}\left(t+t_{d}\right)^{2}}{2}\right\} .
$$

The corresponding eigenvalues are given as $1 / \lambda_{m}=a H_{c} \sqrt{\pi}$ $\sqrt{1-q^{2}} q^{m}$, where $H_{c}=\left|H_{o}(0)\right|^{2} H_{e}(0)$.

We assume the received signal is a Gaussian pulse without the ASE such that

$$
E_{i n}(t)=E_{0} \exp \left\{-b^{2}\left(t+t_{d}\right)^{2} / 2\right\},
$$

where $b$ is a constant. Then its Fourier transform is $\varepsilon_{i n}(\omega)=$ $\sqrt{2 \pi}\left(E_{o} / b\right) \exp \left(j \omega t_{d}-\omega^{2} / 2 b^{2}\right)$ and the 3-dB bandwidth of $\left|E_{i n}(\omega)\right|^{2}$ is $\sqrt{\ln 2} b / \pi$. When $b=a, E_{\text {in }}(t)$ is proportional to $\psi_{0}(t)$. From (7), we find

$$
\begin{aligned}
S_{m}(0) & =\int_{-\infty}^{\infty} \varepsilon_{i n}(\omega) \phi_{m}^{*}(\omega) d \omega \\
& =\frac{2 \pi^{3 / 4} E_{o} \sqrt{a m !}}{2^{m / 2}(m / 2) ! \sqrt{a^{2}+b^{2}}}\left(\frac{b^{2}-a^{2}}{b^{2}+a^{2}}\right)^{m / 2} .
\end{aligned}
$$

In particular, we have for $m=0$

$$
S_{0}(0)=\frac{2 \pi^{3 / 4} E_{o} \sqrt{a}}{\sqrt{a^{2}+b^{2}}} .
$$

The $E_{\text {out }}(t)$ after the optical filter is found as

$$
E_{\text {out }}(t)=\frac{\sqrt{2 \pi} E_{0} A_{0}}{\sqrt{\alpha^{2}+b^{2}}} \exp \left\{-\frac{\alpha^{2} b^{2}\left(t+t_{e}\right)^{2}}{2\left(\alpha^{2}+b^{2}\right)}\right\} .
$$

The photo-detector output current is $(k / 2)\left|E_{\text {out }}(t)\right|^{2}$. The output voltage of the electrical filter can be evaluated exactly as follows:

$$
\begin{aligned}
y(t) & =\frac{k\left|E_{0}\right|^{2} H_{c} a^{2} \sqrt{1-q^{2}}}{2 \sqrt{\left(a^{2}+b^{2}\right)^{2}-q^{2}\left(a^{2}-b^{2}\right)^{2}}} \\
& \cdot \exp \left\{-\frac{(1-q) a^{2} b^{2} t^{2}}{\left(a^{2}+b^{2}\right)-q\left(a^{2}-b^{2}\right)}\right\} .
\end{aligned}
$$

The output waveform of the Gaussian receiver for the Gaussian input (25) is also a Gaussian with its maximum at $t=0$.

From (5), the output of the Gaussian receiver has the following expression:

$$
y(t)=\frac{k a H_{c} \sqrt{1-q^{2}}}{8 \pi^{3 / 2}} \sum_{m=0}^{\infty} q^{m}\left|S_{m}(t)\right|^{2} .
$$

Comparing (30) with (31), we can find the receiver eigenmode contributions at arbitrary times. For example, setting $t=0$, where the output voltage of the Gaussian receiver is maximized, we may expand $y(0)$ obtained from (30) as a Taylor series about the $q=0$ point except the $\sqrt{1-q^{2}}$ factor

$$
\begin{aligned}
y(0)= & \frac{k\left|E_{0}\right|^{2} H_{c} a^{2} \sqrt{1-q^{2}}}{2 \sqrt{\left(a^{2}+b^{2}\right)^{2}-q^{2}\left(a^{2}-b^{2}\right)^{2}}} \\
= & \frac{k}{2}\left|E_{0}\right|^{2} H_{c} \sqrt{1-q^{2}} \frac{a^{2}}{a^{2}+b^{2}} \\
& \cdot\left\{1+\frac{q^{2}}{2}\left(\frac{a^{2}-b^{2}}{a^{2}+b^{2}}\right)^{2}+\frac{3 q^{4}}{8}\left(\frac{a^{2}-b^{2}}{a^{2}+b^{2}}\right)^{4}+\ldots\right\} .
\end{aligned}
$$

Only the even eigenmodes contribute and the lowest-order eigenmode $(m=0)$ contribution becomes dominant when $q$ is much less than 1 . In particular, when $b=a$, only the lowest-order mode term is present at $y(0)$ independent of the $q$ value.

We can find the lowest-order mode contribution to $y(t)$, denoted as $y_{L}(t)$, after setting $q=0$ except the $\sqrt{1-q^{2}}$ factor,

$$
y_{L}(t)=\frac{k\left|E_{0}\right|^{2} H_{c} a^{2} \sqrt{1-q^{2}}}{2\left(a^{2}+b^{2}\right)} \exp \left\{-\frac{a^{2} b^{2} t^{2}}{\left(a^{2}+b^{2}\right)}\right\} .
$$

The ratio between $y_{L}(t)$ and $y(t)$ is

$$
\begin{aligned}
\frac{y_{L}(t)}{y(t)} & =\sqrt{1-q^{2}\left(\frac{a^{2}-b^{2}}{a^{2}+b^{2}}\right)^{2}} \\
& \cdot \exp \left\{-\frac{2 q a^{2} b^{4} t^{2}}{\left(a^{2}+b^{2}\right)\left\{a^{2}+b^{2}-q\left(a^{2}-b^{2}\right)\right\}}\right\} .
\end{aligned}
$$

This ratio has a maximum at $t=0$ where the signal is also maximized. In other words, the contribution of the lowest-order eigenmode is maximized at the pulse center $t$ $=0$. It decreases as $|t|$ departs from zero and higher-order eigenmode contributions increase. The lowest-order eigenmode contribution also decreases as $q$ increases. When $b=a$, we have $y_{L}(t) / y(t)=\exp \left(-q a^{2} t^{2} / 2\right)$. There are no higher-order eigenmode contributions at $t=0$ irrespective of $q$ or $r$.

The correlation functions for the Gaussian optical receiver can be derived as

$$
C_{k m}(t)=2^{m} \sqrt{\pi} \frac{k !}{a}\left(\frac{-a t}{2}\right)^{m-k} L_{k}^{m-k}\left(a^{2} t^{2} / 2\right) \exp \left(-a^{2} t^{2} / 4\right), \quad k \leq m
$$


where $L_{k}^{m-k}(\cdot)$ is the associated Laguerre polynomials [20]. Especially, when $k=0$, we have

$$
C_{0 m}(t)=j^{-m} \sqrt{\frac{2^{m}}{m !}}\left(-\frac{a t}{2}\right)^{m} \exp \left(-a^{2} t^{2} / 4\right) .
$$

When $b=a$, (30) reduces to

$$
y(t)=\frac{k\left|E_{0}\right|^{2} H_{c} \sqrt{1-q^{2}}}{4} \exp \left\{-\frac{(1-q) a^{2} t^{2}}{2}\right\} .
$$

Comparing (38) with (31), we find

$$
\begin{aligned}
\left|S_{m}(t)\right|^{2} & =\frac{2 \pi^{3 / 2}}{a}\left|E_{0}\right|^{2} \frac{\left(a^{2} t^{2} / 2\right)^{m}}{m !} \exp \left(-a^{2} t^{2} / 2\right) \\
& =\left|S_{0}(0)\right|^{2} \frac{\left(a^{2} t^{2} / 2\right)^{m}}{m !} \exp \left(-a^{2} t^{2} / 2\right)
\end{aligned}
$$

This result matches with (37).

In Fig. 1(a), we plot $y(t)$ and $y_{L}(t)$ for $r=2(q=0.268)$ for several $b$ values. $y(t)$ and $y_{L}(t)$ are normalized by $y_{d c}$ which is the dc response of $y(t)$ with $b=0$. Between the two traces for a given $\mathrm{b}$ value, the lower one corresponds to $y_{L}(t)$. At $b=0$, the signal is unmodulated and about $4 \%$ of $y(t)$ is the contributions from higher-order eigenmodes. As $b$ increases from zero to $a$, the higher-order eigenmode contributions decrease to zero at $t=0$, where the signal power is maximized. When $b=a$, only the lowest-order eigenmode contributes or $y(0)=y_{L}(0)$ As $b$ increases further from $a$, the contributions of higher-order eigenmodes increase at $t=0$ but more slowly. In Fig. 1(b), we use $r=4(q=0.5)$. At $b=0$, about $13 \%$ of $y(t)$ belongs to higher order eigenmodes. Overall, the higher-order eigenmode contributions are larger than Fig. 1(a). When $b=a$, only the lowest-order eigenmode contributes at $t=0$ as Fig. 1(a).

In Fig. 2(a), we show the pdfs of $\bar{y}(t)=y(t) / y_{s p}$ for $b=a$ and for $r=2(q=0.268)$ at $t=0$ and $T / 4 . T$ is the bit period chosen to be 100 ps assuming the bit rate as 10 $\mathrm{Gb} / \mathrm{s} . y_{s p}$ is the average value of $y(t)$ per polarization in the absence of the signal given as $\left(k \sigma_{\omega}^{2} / 4 \pi^{2}\right) \sum_{m=0}^{\infty} \lambda_{m}^{-1}$ $=k a H_{c} \sigma_{\omega}^{2} \sqrt{1+q} / 4 \pi^{3 / 2} \sqrt{1-q}$. We have set $A_{0}(0)=81.3$ and $A_{m \neq 0}$ that gives $10^{-9}$ bit-error rate at $t=0$ [14]. The pdfs are found as an inverse Fourier transform of the characteristic function of $y(t)$ [10]. We have used 30 eigenmodes in the full eigenmode analysis (FEA) and used only the lowest-order eigenmode for the single-eigenmode analysis (SEA). We have chosen $\sqrt{\ln 2} \beta / 2 \pi$ as $7 \mathrm{GHz}$. At $t=0$, the FEA and the SEA give almost the same pdfs, which means the effects from $\left\{N_{m \neq 0}(0)\right\}$ are negligible. At $t=T / 4$, the FEA gives more symmetric pdf with respect to its peak than that of the SEA. Note that the pdf at $t=0$ is more symmetric than the pdf obtained by the FEA at $t=$ $T / 4$. We show in Fig. 2(b) the pdfs of $\bar{y}(t)$ for $r=4$ $(q=0.5)$ with all other conditions the same as Fig. 2(a). In this case, as Fig. 1 shows, higher order eigenmodes become more important at $t=T / 4$ than Fig. 2(a). At $t=0$, the FEA and the SEA still give almost the same pdfs. At $t=T / 4$, the FEA gives more symmetric pdf than the SEA. The pdf at $t=0$ has a similar symmetry with respect to its peak as the pdf obtained by the FEA at $t=T / 4$.

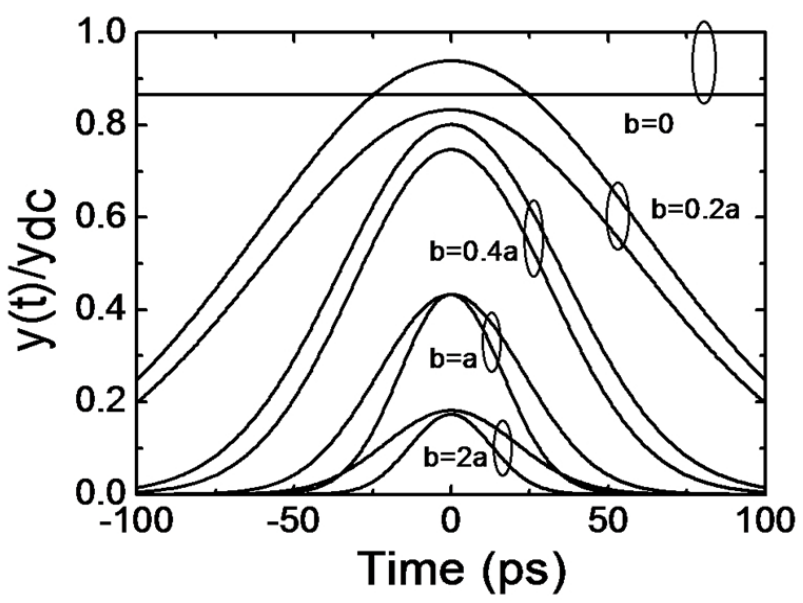

(b)

FIG. 1. The traces of $y(t)$ and $y_{L}(t)$ normalized by $y_{d c}$ which is the dc response of $y(t)$ with $b=0 . y_{L}(t)$ is obtained from $y(t)$ neglecting all the eigenmode contributions except $m=0$. For each value of $b$, the upper trace is $y(t) / y_{d c}$ and the lower trace is $y_{L}(t) / y_{d c}$. (a) $r=2$ $(q=0.268)$. (b) $r=4(q=0.5)$. 


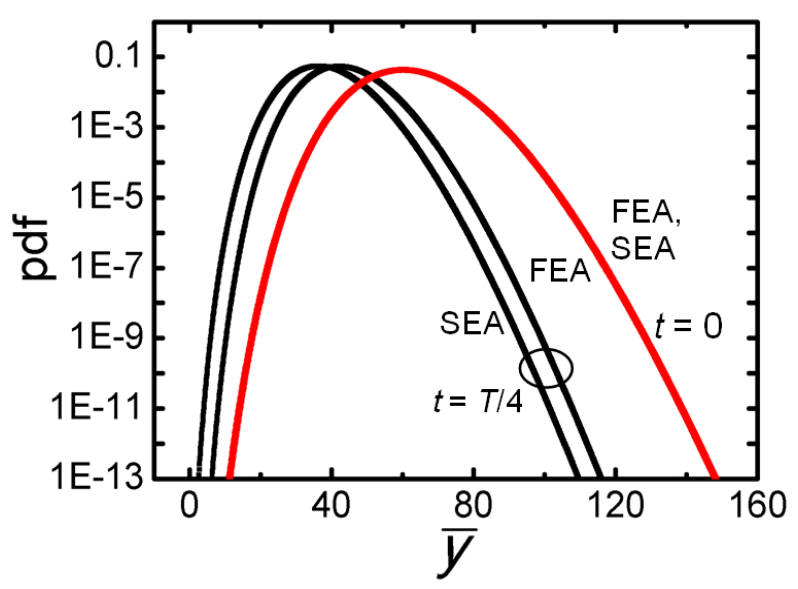

(a)

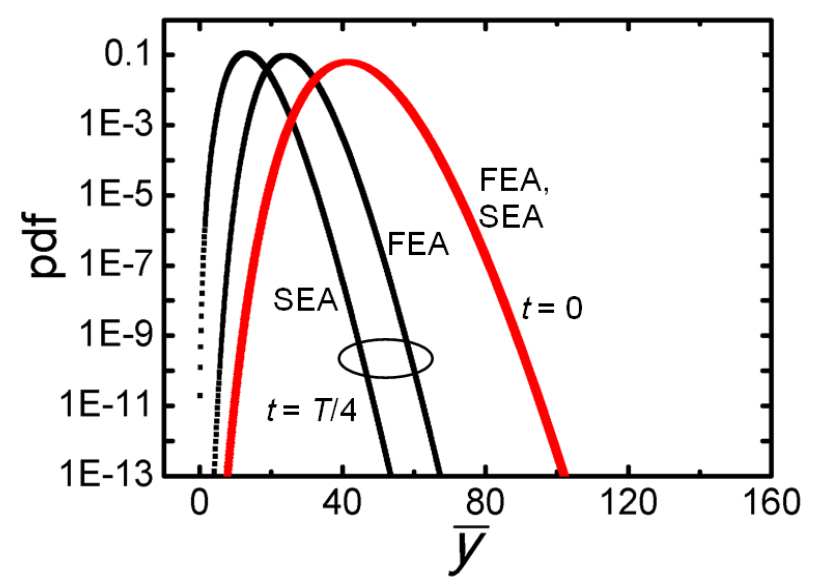

(b)

FIG. 2. Probability density functions of $\bar{y}(t)=y(t) / y_{s p}$ at $t=0$ and at $t=T / 4$, where $T$ is the bit period which is equal to 100 ps. FEA: full-eigenmode analysis using 30 receiver eigenmodes. SEA: single-eigenmode analysis using the lowest-order receiver eigenmode. (a) $r=2(q=0.268)$. (b) $r=4(q=0.5)$.

\section{CONCLUSION}

We have analyzed the time-dependent behaviors of optical receivers corrupted by the ASE using receiver eigenmodes. The correlation functions between receiver eigenmodes in time domain determine the amplitudes of eigenmodes at different times. Gaussian optical receivers are used for our analysis that have Gaussian optical and electrical filters with closed-form receiver eigenfunctions. The edge parts of the Gaussian electrical pulse have larger contributions of higher-order eigenmodes than the pulse center where the lowest-order eigenmode is dominant. This effect is dependent on the bandwidth ratio between the optical and the electrical filters as well as the input pulse's time width. It is enhanced when the time-width of the received Gaussian optical pulse decreases or when the bandwidth ratio increases. According to the central limit theorem, as the number of contributing eigenmodes increase, the voltage distribution of the electrical pulse at that instant becomes more symmetric and close to Gaussian. Our analysis explains the time-dependent signal and noise properties within the electrical pulses at the output of the receiver electrical filter. For Gaussian optical receivers, we have shown that the best decision time is at the peak of the electrical pulse. For other cases of non-Gaussian filters and of non-Gaussian input pulses, our analysis can be used also to get the best decision timing.

\section{ACKNOWLEDGMENT}

This research was supported partly by the Basic Science Research Program through the National Research Foundation of Korea (NRF) funded by the Ministry of Education, Science, and Technology (2013R1A1A2012918) and also partly supported by the Research Grant of Kwangwoon University in 2012.

\section{REFERENCES}

1. S. D. Personick, "Statistics of a general class of avalanche detectors with applications to optical communication," Bell Syst. Tech. J. 50, 3075-3096 (1971).

2. S. D. Personick, "Receiver design for digital fiber optic communications systems, I," Bell Syst. Tech. J. 52, 843-874 (1973).

3. T. Okoshi and K. Kikuchi, Coherent Optical Fiber Communications (KTK Scientific Publishers (KTK), Tokyo, Japan, 1988).

4. N. A. Olson, "Lightwave systems with optical amplifiers," J. Lightwave Technol. 7, 1071-1082 (1989).

5. B. W. Kang and C. H. Kim, "Performance evaluation of bidirectional optical amplifiers for amplified passive optical network based on broadband light source seeded optical sources," J. Opt. Soc. Korea 15, 4-8 (2011).

6. M. Kac and A. J. F. Siegert, "On the theory of noise in radio receivers with square law detectors," J. Appl. Phys. 18, 383-397 (1947).

7. R. C. Emerson, "First probability densities for receivers with square law detectors," J. Appl. Phys. 24, 1168-1176 (1953).

8. J. E. Mazo and J. Salz, "Probability of error for quadratic detectors," Bell Syst. Tech. J. 44, 2165-2186 (1965).

9. G. Bosco, A. Carena, V. Curri, R. Gaudino, P. Poggiolini, and S. Benedetto, "A novel analytical method for the BER evaluation in optical systems affected by parametric gain," IEEE Photon. Technol. Lett. 12, 152-154 (2000).

10. J. S. Lee and C. S. Shim, "Bit-error-rate analysis of optically preamplified receivers using an eigenfunction expansion method in optical frequency domain," J. Lightwave Technol. 12, 1224-1229 (1994). 
11. P. J. Winzer, M. Pfennigbauer, M. M. Strasser, and W. R. Leeb, "Optimum filter bandwidths for optically preamplified NRZ and RZ receivers," J. Lightwave Technol. 19, 1263-1273 (2001).

12. R. Holzlohner, V. S. Grigoryan, C. R. Menyuk, and W. L. Kath, "Accurate calculation of eye diagrams and bit error rates in optical transmission systems using linearization," $\mathrm{J}$. Lightwave Technol. 20, 389-400 (2002).

13. E. Forestieri and M. Secondini, "On the error probability evaluation in lightwave systems with optical amplification," J. Lightwave Technol. 27, 706-717 (2009).

14. J. S. Lee and A. E. Willner, "Analysis of Gaussian optical receivers," J. Lightwave Technol. 31, $2987-2993$ (2013).

15. S. Y. Kim, K. H. Seo, and J. S. Lee, "Spectral efficiencies of channel-interleaved bidirectional and unidirectional ultradense WDM for metro applications," J. Lightwave Technol. 30, 229-233 (2012).
16. B. Batsuren, H. H. Kim, C. Y. Eom, J. J. Choi, and J. S. Lee, "Optical VSB filtering of $12.5-\mathrm{GHz}$ spaced $64 \times 12.4$ Gb/s WDM channels using a pair of Fabry-Perot filters," J. Opt. Soc. Korea 17, 63-67 (2013).

17. D. Marcuse, "Derivation of analytical expressions for the bit-error probability in lightwave systems with optical amplifiers," J. Lightwave Technol. 8, 1816-1823 (1990).

18. P. A. Humblet and M. Azizoglu, "On the bit error rate of lightwave systems with optical amplifiers," J. Lightwave Technol. 9, 1576-1582 (1991).

19. A. Papoulis, Probabiliry, Random Variables, and Stochastic Processes, 4th ed. (McGraw-Hill, New York, USA, 2002), Chapter 7.

20. G. B. Arfken and H. J. Weber, Mathematical Methods for Physicists, 6th ed. (Elsevier Academic, New York, USA, 2005), Chapter 13. 